\title{
MODEL NUCLEAR MATTER CALCULATIONS WITH A NEW FERMTON LOWEST ORDER CONSTRAINED VARIATIONAL METHOD
}

\author{
J. C.OWEN \\ Department of Theiretical Phyidics, Untoerdty of Menchester, Mancheste MIS SPL, UK \\ and \\ R. F. BISHOP and J. M. IRVINE \\ Department of Theoretical Physics, Undwaity of Manchester, Manchester MI3 SPL, UK \\ and \\ Darabury Laboratory, Sclence Reveanal Cownell, Darabury, Wamington WA4 4AD, UK
}

Recoived 13 May 1976

(Revied 9 July 1976)

\begin{abstract}
Abetract: We extond to deal with formions a mothod of lowest onder contrained variation for calculating the ground-otate energy of dense system, advanced provioubly by the prewent authors and shown to give excellent apreement for the Bethe bomework problem. Uaing one state-independent corrolation function in the new formalism, resultu with four different potentials that model various aspects of the real NN force aro shown to bo in excellent agreement with the other result available from various highor-order approximations, for densities up to and beyond nuclear matter denaity. The arreement is clearly superior to that obtainod with any other lowest order approximation. When state dopendence is introduced into the two-body correlation function in the now method, we find a condiderable lowerins of the onores in each case. It is sugseated that thin is a real offect which would be roplicated in the other higher-orior approximations, were they also extended to deal with state-dependent correlations.
\end{abstract}

\section{Introduction}

Several publications by a number of authors ${ }^{1-4}$ ) have appeared recently, which calculate the energy of infinite, strongly interacting model Fermi systems using the method of Jastrow correlation functions, and which involve the explicit summation of many of the higher order terms in the cluster series. These calculations all tend to be difficult and lengthy, and it seems that for applications to real physical systems such as nuclear matter or finite nuclei with realistic potentials, there is still a demand for a reliable low order approach. To this end we generalize in sect. 2 a method of lowest order constrained variation previously presented by the present authors ${ }^{5-7}$ ), and impose new constraints which seem suited to dense Fermi systems.

We compare results obtained by our method with the hypernetted chain (HNC) calculations of Pandharipande $e t$ al. ${ }^{1}$ ) for two model potentials, namely the ${ }^{1} S_{0}$ part and the central part of the ${ }^{3} S_{1}-{ }^{3} D_{1}$ Reid soft core potential ${ }^{8}$ ), for both Fermi and Bose systems, and also with the fermion hypernetted chain (FHNC) and the fermion Percus-Yevick (FPY) calculations of Campani et al. ${ }^{2}$ ) for the 
model hard core IY [ref. ${ }^{9}$ )] and OMY [ref. ${ }^{10}$ )] potentials. The overall agreement between these calculations, each of which uses a state-independent correlation function, is extremely encouraging and suggests that a simple lowest order method as presented here is perfectly capable of providing reasonably reliable results, at least up to nuclear matter densities, for a wide range of central potentials.

We also introduce state dependence into our correlation functions, and find that this generally results in a considerable lowering of the energy; typically $\approx 5 \mathrm{MeV}$ at nuclear matter densities. For the IY and OMY potentials, where comparison can be made, this brings our results into apparent agreement with the recent calculations of Clark et al. ${ }^{3}$ ), which omploy state-independent correlation functions but further introduce a second-order perturbation correction to the energy via the method of correlated basis functions.

\section{Method}

We consider a system of $N$ identical particles interacting pairwise through a twobody central potential $V(r)$, each of mass $m$, and enclosed in a volume $Q$, in the thermodynamic limit $N \rightarrow \infty, \Omega \rightarrow \infty$ such that the density $\rho=N / \Omega$ remains finite.

For bosons we consider the uncorrelated ground state to be with all the particles in the zero momentum condensate, and the uncorrelated relative two-body wave function $\phi / \Omega$ is given by

$$
r^{2} \phi^{2}(r)=a^{2}(r)=r^{2}(1-1 / N) .
$$

In the case of fermions we consider the uncorrelated ground state to be the filled Fermi sea characterised by a Fermi momentum $k_{\mathrm{F}}=\left(6 \pi^{2} \rho / v\right)^{\frac{t}{t}}$, where $v$ is the fermion spin-isospin degeneracy factor. As appropriate, the potential and the two-body correlation function, both of which are considered momentum independent, can be decomposed into relative partial wave components $V_{l}(r)$ and $f_{l}(r)$ respectively. After averaging over the Fermi sea, the uncorrelated fermion wave function $\phi(r)$ may be expressed as

$$
r^{2} \phi^{2}(r)=r^{2} \sum_{l} \phi_{l}^{2}\left(k_{\mathrm{F}} r\right) P_{l}=\sum_{l} a_{l}^{2}(r) P_{l}
$$

where $P_{l}=|l\rangle\langle l|$ is the projector on to the $l$ th partial wave. If the potential and two-body correlation function are both state independent, the sum over partial waves in eq. (2) may be performed analytically to obtain,

$$
r^{2} \phi^{2}(r)=r^{2}\left[1-\frac{9}{v} \frac{j_{1}^{2}\left(k_{\mathrm{F}} r\right)}{\left(k_{\mathrm{F}} r\right)^{2}}\right],
$$

where $j_{1}$ is the usual spherical Bessel function.

In previous publications ${ }^{5,6}$ ) we have described a method of lowest order constrained variation (LOCV) which involves truncation of the Jastrow cluster series at the two-body term, but with the imposition of constraints on (the partial-wave 
components of) the two-body correlation function $f(r)$ which are devised to justify this truncation. The constraints have been formulated so that $f_{i}(r)$ should both satisfy a certain bound of the form,

$$
0 \leqq f_{l}(r) \leqq h_{l}(r),
$$

and, simultaneously, satisfy an integral inequality of the form,

$$
\sum_{l} 4 \pi \rho \int_{0}^{\infty} G_{l}\left[f_{i}(r), h_{l}(r)\right] \mathrm{d} r \leqq I,
$$

where the functional $G_{l}$ satisfies the condition,

$$
G_{l}\left[h_{r}(r), h_{l}(r)\right] \equiv 0 .
$$

We have shown that, at least for realistic potentials $V_{l}(r)$ and specified functions $h_{l}(r)$, minimization of the two-body energy in the cluster expansion,

$$
E_{2} / N=2 \pi \rho \sum_{l} \int_{0}^{\infty}\left[h^{2} m^{-1} f_{l}^{\prime 2}(r)+V_{l}(r) f_{l}^{2}(r)\right] a_{l}^{2}(r) d r,
$$

subject to the constraints (4)-(6), is achieved as follows. The solutions to each of the Euler-Lagrange equations,

$$
\begin{gathered}
g_{l}^{\prime \prime}(r)-\left\{\frac{a_{l}^{\prime \prime}(r)}{a_{l}(r)}+\frac{m}{\hbar^{2}} V_{l}(r)\right\} g_{l}(r)+\frac{m \lambda}{2 \hbar^{2} a_{l}(r)} \frac{\delta G_{l}}{\delta f_{l}}=0, \\
g_{l}(r) \equiv a_{l}(r) f_{l}(r),
\end{gathered}
$$

is first obtained in the range $0 \leqq r \leqq d_{l}$, where the correlation distances $d_{l}$ are defined by $f_{l}^{\prime}\left(d_{l}\right)=h_{l}^{\prime}\left(d_{l}\right)$; and for $r>d_{l}$ we set $f_{l}(r) \equiv h_{l}(r)$. The constant $\lambda$ in eq. (8), which is the Lagrange multiplier for constraint (5), is either set to zero if this constraint is thus satisfied as an inequality, or it is varied iteratively until it is satisfied as an equality. It is easy to show that after this minimization procedure has been performed, the two-body energy of eq. (7) can be re-expressed in the form,

$$
\begin{aligned}
\frac{E_{2}}{N}=2 \pi \rho \sum_{l}\left\{\frac{1}{2} \lambda \int_{0}^{d_{1}} \mathrm{~d} r f_{l}(r) \frac{\delta G_{1}}{\delta f_{l}}\right. & +\int_{d_{l}}^{\infty} \mathrm{d} r a_{l}^{2}(r) \\
\times & {\left.\left[V_{l}(r) h_{l}^{2}(r)-\frac{h^{2}}{m} h_{l}(r)\left(h_{l}^{\prime \prime}(r)+\frac{2 h_{l}^{\prime}(r) a_{l}^{\prime}(r)}{a_{l}(r)}\right)\right]\right\} . }
\end{aligned}
$$

For Fermi systems, the total energy also contains a one-body kinetic energy contribution of $\frac{3}{10} \hbar^{2} k_{\mathrm{F}}^{2} / m$, which is included in all numerical results quoted in sect. 3 .

In all previous work ${ }^{5-7}$ ) we have presented arguments to use the above constraints in the form,

$$
\begin{gathered}
h_{l}(r)=1, \\
G_{l}\left[f_{l}, h_{l}\right]=\left[h_{l}^{2}(r)-f_{l}^{2}(r)\right] a_{l}^{2}(r)=\left(1-f_{l}^{2}(r)\right) a_{l}^{2}(r), \\
I=1,
\end{gathered}
$$


where the subscript $l$ is only appropriate for fermions, and calculations have been performed for this set of constraints. For Bose systems we have justified constraint (10) on the grounds that in a two-body approximation it is unreasonable to allow the attractive tail of the typical physical potential to have a large effect on the form of the correlation function. Similarly constraints (11) and (12) have been motivated on the grounds that for consistency within a two-body approximation, the introduction of correlations should not be allowed to expel more than one particle from within the range of strong influence of another, i.e. from within the correlation distance. These constraints were previously ${ }^{5}$ ) found to give excellent agreement with other more detailed calculations involving explicit evaluation of higher order terms for Bose systems interacting via the repulsive part of the Reid soft core ${ }^{1} \mathbf{S}_{0}$ potential in all partial waves (the so-called Bethe homework problem). Good agreement is also found with these constraints for Bose systems interacting via the model potentials used here, as described later in sect. 3.

On the other hand, for Fermi systems, where the Pauli exclusion principle itself induces short-range correlations in the system even in the absence of interactions, and thereby effects the magnitude of higher order terms in the cluster series for the energy, such average arguments as were used to justify constraints (10)-(12) carry less weight, and these constraints may be considered less appropriate. In particular, for Fermi systems, the factor $a^{2}(r)\left[=\left\{1-9 v^{-1}\left(j_{1}\left(k_{\mathrm{F}} r\right) / k_{\mathrm{F}} r\right)^{2}\right\} r^{2}\right.$, assuming stateindependent correlation functions and performing the sum over $l]$ which appears in eq. (11) is depressed near the origin in comparison with its Bose counterpart $(1-1 / N) r^{2}$. Hence, constraint (5) becomes a weaker constraint for fermions than for bosons, which may result in rather poor constraining of the Fermi correlation function, particularly at higher densities. We could of course compensate for this effect simply by depressing the value of the constant $I$ in eq. (5) for fermions to below unity, whilst keeping $I=1$ for bosons. Although this may well be appropriate in some cases (and will form the basis of a later publication), it seems more natural at present to attempt to reformulate eqs. (10) and (11) on more general grounds.

We first note that for a Bose system, the minimum value of $E_{2} / N$ as given by eq. (7) or (9) is changed only by $O(1 / N)$ if eq. (10) is replaced by,

$$
h^{2}(r) \phi^{2}=h^{2}(r)(1-1 / N) \equiv 1,
$$

and correspondingly eq. (11) is changed to read,

$$
G[f, h]=\left[h^{2}(r)-f^{2}(r)\right] r^{2} \phi^{2}=\left\{1-f^{2}(r) \phi^{2}\right\} r^{2},
$$

in order to satisfy eq. (6). Constraint (5) then takes the form,

$$
\rho \int_{0}^{\infty}\left\{1-f^{2}(r) \phi^{2}\right\} \mathrm{d} r \leqq I .
$$

If we take $I=1$ as before, and identify $f^{2}(r) \phi^{2}$ as the two-body approximation to the two-body radial distribution function, then eq. (15) reckoned as an equality 
merely expresses the normalization condition ${ }^{11}$ ) which must be satisfied by any exact radial distribution function. Whilst this may be considered to be a purely formal procedure since now the correlations have a long-range component, albeit of $O(1 / N),\left[f^{2}=(1-1 / N)^{-1} ; r>d\right]$, it may also provide an indication of why constraints (10)-(12) give such good results for bosons for the various model potentials considered, since it is known that imposition of the normalization condition has the effect of stimulating rapid convergence of the cluster series ${ }^{12}$ ). Employing a similar procedure for Fermi systems leads us by analogy to the choice,

or equivalently,

$$
h_{l}^{2}(r) \phi^{2}(r)=1
$$

$$
f_{l}(r)=\left[1-\frac{9}{v}\left(\frac{j_{1}\left(k_{\mathrm{F}} r\right)}{k_{\mathrm{F}} r}\right)^{2}\right]^{-t} ; \quad r>d_{1}
$$

Again, the constraint (5) is simultaneously chosen (with $I=1$ ) as

$$
\rho \int_{0}^{\infty}\left[1-\sum_{l} f_{l}^{2}(r) \phi_{l}^{2}(r)\right] \mathrm{d} r \leqq 1,
$$

or equivalently as,

$$
\rho \sum_{l} \int_{0}^{\infty}\left[\left\{1-\frac{9}{v}\left(\frac{j_{1}\left(k_{\mathrm{F}} r\right)}{k_{\mathrm{F}} r}\right)^{2}\right\}^{-1}-f_{l}^{2}(r)\right] \phi_{l}^{2}(r) \mathrm{d} r \leqq 1 .
$$

For comparison with eqs. (10)-(12), the reformulated constraints now take the form,

$$
\begin{gathered}
h_{l}(r)=\left\{1-\frac{9}{v}\left(\frac{j_{1}\left(k_{\mathrm{F}} r\right)}{k_{\mathrm{F}} r}\right)^{2}\right\}^{-t}, \\
G_{l}\left[f_{l} h_{l}\right]=\left[h_{l}^{2}(r)-f_{l}^{2}(r)\right] a_{l}^{2}(r)=\left[\left\{1-\frac{9}{v}\left(\frac{j_{1}\left(k_{\mathrm{F}} r\right)}{k_{\mathrm{F}} r}\right)^{2}\right\}^{-1}-f_{l}^{2}(r)\right] a_{l}^{2}(r) . \\
I=1 .
\end{gathered}
$$

Eq. (18) shows the constraint in the form of the normalization condition (assuming equality); and eq. (19), or equivalently eq. (21), shows that $G_{l} \equiv 0$ for $f_{l}(r)=h_{l}(r)$ as required. It is also worth noting at this point that a simple rearrangement of eq. (19), together with the relation,

$$
\int_{0}^{\infty} d x x^{-4}(\sin x-x \cos x)^{2}=\frac{1}{6} \pi
$$

shows that the convergence parameter ${ }^{13}$ ) $\kappa$ associated with the cluster expansion,

$$
\kappa=\rho \sum_{l} \int \operatorname{dr}\left[1-f_{l}^{2}(r)\right] \phi_{l}^{2}(r)
$$

is identially zero when the equality sign is taken is taken in eq. (19). 
The choice of eq. (16) to describe the long-range behaviour of $f_{l}(r)$ may perphaps seem somewhat arbitrary at first sight, but it does appear to be forced upon us if we require that the integral constraint should take the form of the normalization condition and that for bosons it should reduce to the form of eqs. (10)-(12). The longrange behaviour of the correlation functions $f_{l}(r)$, which is dictated by the function $h_{l}(r)$, is characterized with the choice of eq. (20) by an "overshool", although this is typically small and of quite short range. Thus, for example, $h_{l}(r) \approx 1.05$ at $r=r_{\mathrm{c}}$, the characteristic or unit radius (defined by $\rho^{-1}=\frac{4}{3} \pi r_{0}^{3}$ ), for fermions of degeneracy $v=4$. The overshoot is of even shorter range for $v=2$. It seems that the principle effect at high densities of using constraints (4) - (6) with the form of eqs. (20)-(22) rather than (10)-(12), is to blunt the effect that the factor $a_{l}^{2}(r)$ in eq. (11) has in reducing the value of the integral in eq. (5). At low densities the short-ranged overshoot thus allowed in the correlation function should clearly also be beneficial. Finally we note that it is appropriate to leave constraint (18) [i.e. equivalently eqs. (5) and (21)] as an inequality, rather than the equality that strict imposition of the normalization condition dictates, since it is surely not sensible to force a larger short-range wound into the correlation function than is required by the potential itself.

Clearly there are many other possible choices which we could have made for the functions $h_{l}(r), G_{l}$ and $I$. Thus, for example, we could follow the work of Băckman et al. ${ }^{13}$ ) and parametrize the long-range overshoot behaviour $h_{l}(r)$ of the correlation function by some predetermined functional form, and fix the functional $G_{l}$ of eq. (5) to express it as the orthogonality condition. Finally, a parameter search for best values to use in the particular functional form chosen for $h_{l}(r)$ which minimize the two-body energy, could be undertaken. [Note that a free variation of the function $h_{l}(r)$ gives meaningless results, due to the "Emery difficulty" ${ }^{4}$ ), even when a constraint such as eq. (5) is employed.] In fact, we have chosen to use the two-body approximation to the normalization condition as our principal integral constraint, and to use the simplest possible long-range behaviour for the correlation function which is compatible with this constraint, as outlined above. In defence of this procedure we stress once again that for bosons this choice is completely equivalent to the choice of constraints (10)-(12) which we have used in previous work. Our philosophy has been that since these constraints can both be justified from simple physical arguments, and have previously been shown to be so successful in describing such simple models as the "Bethe homework problem", they are likely to prove worthy of the further refinement and application to Fermi systems as already described.

In the following section we describe results obtained by our method of lowest order constrained variation, for both Bose and Fermi systems, and employing constraints (4)-(6) both with the choices expressed in eqs. (10)-(12) as well as in eqs. (20)-(22). We shall refer to these constraints respectively as boson constraints and normalization constraints and shall accordingly label the method we have outlined which employs them as LOCVB and LOCVN respectively. The two methods of course become identical for Bose systems. 


\section{Results}

We have performed calculations of the ground-state energy per particle, $E / N$, for systems interacting via any of four model potentials which have been widely used by previous authors, and with which comparisons can be made. Two of these potentials have been used previously by Pandharipande et al. ${ }^{1}$ ) among others, and are drawn from the Reid soft core potential ${ }^{8}$ ); and are respectively the potential in the ${ }^{1} S_{0}$ channel, and the central part only of the potential in the ${ }^{3} S_{1}-{ }^{3} D_{1}$ channel, each considered as a local potential acting in all states. The other two model potentials considered are those of Iwamoto-Yamada ${ }^{9}$ ) and Ohmura-Morita-Yamada ${ }^{10}$ ), known in the literature as IY and OMY respectively. We denote these potentials respectively by V1, V2, V3 and V4. Whilst V1 and V2 are soft-core potentials, V3 and V4, as used here, both contain a state-independent hard core of radius $0.6 \mathrm{fm}$ in all states. Both V3 and V4 have zero attraction in odd-parity states. [Note that in their original forms of refs. ${ }^{9,10}$ ) V3 and V4 are considered to be pure Serber exchange potentials, i.e. strictly zero in all odd waves, but for the sake of comparison with other calculations, we consider the hard core to be present in all partial waves.] The potential V3 contains a spin-independent attraction in even states, and V4 is identical with V3 in spin-singlet even states, but has a different attractive part for spin-triplet even states. Each of the four potentials clearly models some aspects of the real internucleon force. We show a plot of the four potentials in fig. 1, and give their analytic

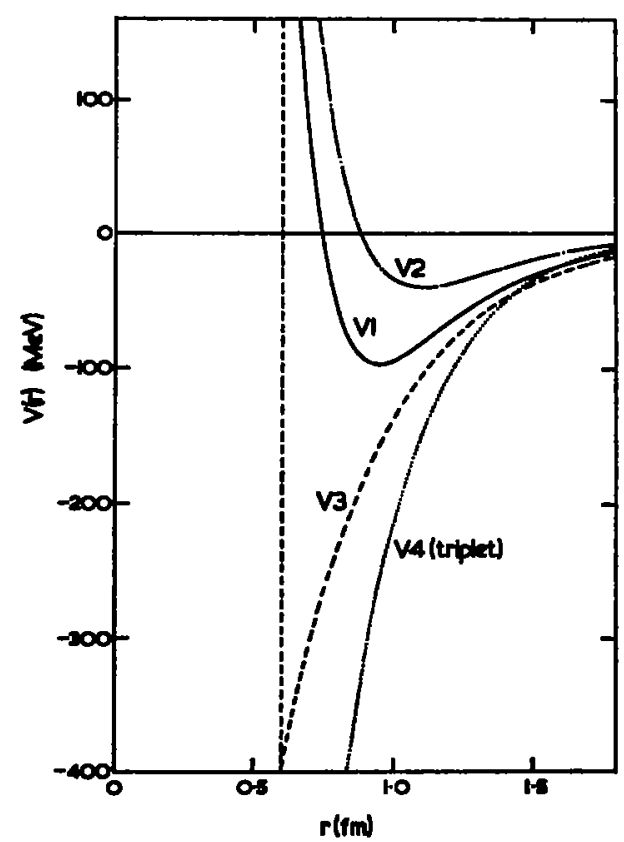

Fig. 1. The four model potentials used in this work. 
forms below, each in units of $\mathrm{MeV}$ when $r$ is expressed in fm:

$$
\begin{aligned}
V_{1}(r) & =-10.463 e^{-x} / x-1650.6 e^{-4 x} / x+6484.2 e^{-7 x} / x, \\
V_{2}(r) & =-10.463 e^{-x} / x+105.468 e^{-2 x} / x-3187.8 e^{-4 x} / x+9924.3 e^{-6 x} / x, \\
x & =0.7 r ; \\
V_{3}(r) & = \begin{cases}\infty, & r<0.6 \\
-397.3 e^{-2.627(r-0.6)} P_{+}, & r>0.6,\end{cases} \\
V_{4}(r) & = \begin{cases}\infty, & r<0.6 \\
-397.31 e^{-2.6272(r-0.6)} P_{+} P_{s}-947.02 e^{-3.6765(r-0.6)} P_{+} P_{t}, & r>0.6,\end{cases}
\end{aligned}
$$

where $P_{+}$is the projector on to even partial waves, $P_{1}=\frac{1}{4}\left(1-\sigma_{1} \cdot \sigma_{2}\right)$ is the spinsinglet projector, and $P_{\mathrm{t}}=\frac{1}{4}\left(3+\sigma_{1} \cdot \sigma_{2}\right)$ is the spin-triplet projector.

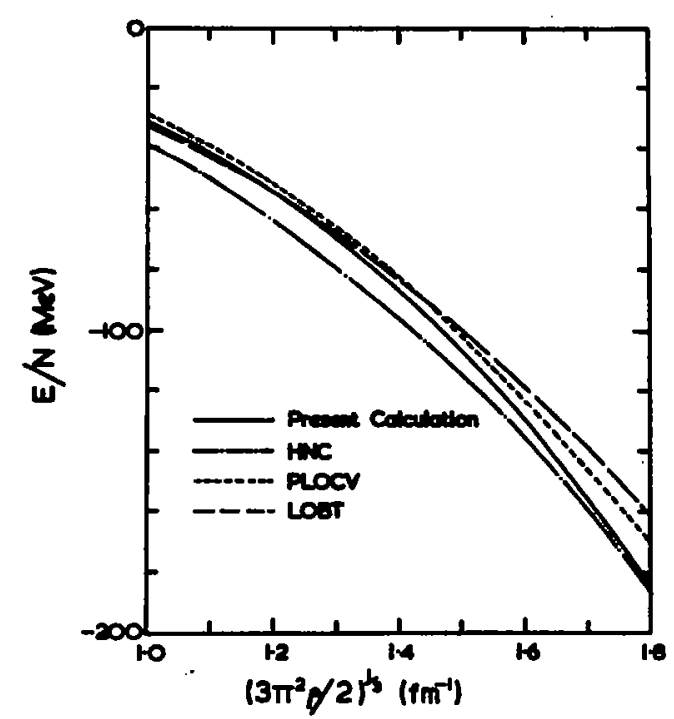

Fig. 2. The ground-state energy per particle for a Bose system with poteniial V1. The results uning the hypernotted chain approximation (HNC), Pandharipando's lowest order constrainod variation (PLOCV), and loweat order Bruecknor theory (LOBT), are taken from ref. ${ }^{1}$ ).

In figs. 2 and 3 we display the results for potentials V1 and V2 for a system of bosons. We compare our results with calculations on the same systems taken from ref. ${ }^{1}$ ) using: (i) the hypernetted chain approximation to the exact two-body distribution function (HNC); (ii) the method of lowest order Brueckner theory (LOBT); and (iii) Pandharipande's method of lowest order constrained variation (PLOCV). The choice of abscissa in figs. 2 and 3 is made in order to facilitate comparison with fermions of degeneracy $v=4$, which form the subject of the remaining figures. 


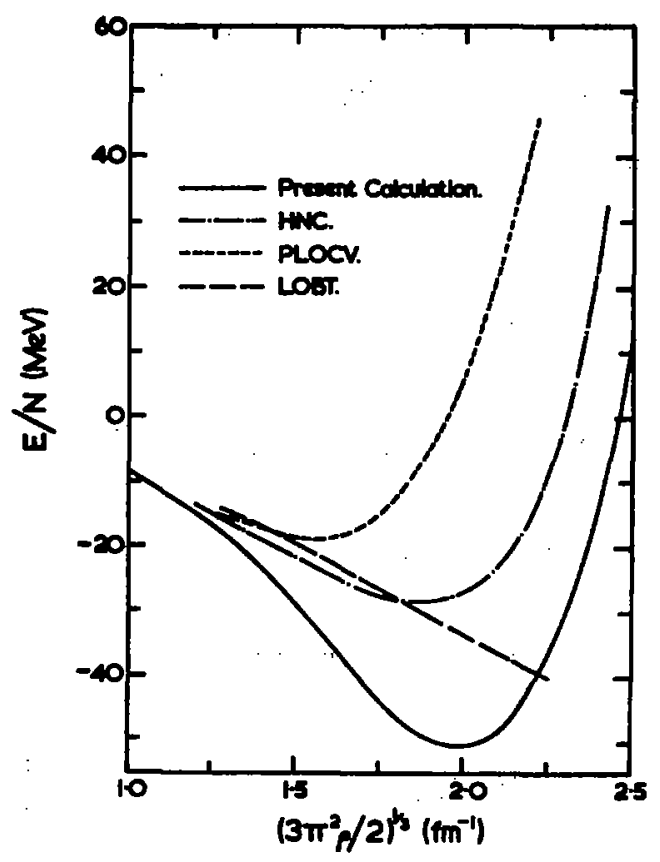

Fig. 3. Same as fig. 2, but with the potential $\mathbf{V}$

Tane 1

Values of the Lagrange multiplier, $\lambda$ (in MoV), associated with the normalization integral constraint, for the four modal potentials

\begin{tabular}{|c|c|c|c|c|c|c|c|c|c|}
\hline \multirow{3}{*}{$\begin{array}{l}\left(f \pi^{2} \rho\right) t \\
\left(\mathrm{fm}^{-1}\right)\end{array}$} & \multicolumn{2}{|c|}{ V1 } & \multicolumn{3}{|c|}{$\mathbf{V} 2$} & \multirow{2}{*}{\multicolumn{2}{|c|}{$\frac{\text { V3 }}{\text { Fermi }}$}} & \multirow{2}{*}{\multicolumn{2}{|c|}{$\begin{array}{l}\text { V4 } \\
\text { Fermi }\end{array}$}} \\
\hline & \multirow[t]{2}{*}{ Boso } & \multirow{2}{*}{$\begin{array}{l}\text { Formi } \\
\text { N1 *) }\end{array}$} & \multirow[t]{2}{*}{ Bose } & \multicolumn{2}{|c|}{ Fermi } & & & & \\
\hline & & & & N1 & $\overline{\left.\mathrm{N} 2^{\circ}\right)}$ & N1 & N2 & Ni & N4") \\
\hline 1.2 & 0 & 0 & 0 & 0 & 0 & 0 & -0.36 & 0 & -0.29 \\
\hline 1.4 & 0 & 0 & 0 & 0 & 0 & 0 & -2.69 & 0 & -1.41 \\
\hline 1.6 & 0 & 0 & -1.95 & -6.71 & -10.9 & -6.28 & -16.6 & 0 & -6.58 \\
\hline 1.8 & 0 & 0 & -34.9 & -42.4 & -47.4 & -95.8 & -97.8 & -52.8 & -69.9 \\
\hline 2.0 & 0 & 0 & -104 & -113 & -120 & -446 & -446 & -366 & -378 \\
\hline
\end{tabular}

c) The notation N1, N2 and N4 for Fermi systems indicates the number of independent corrolation functions.

We note that the relatively small effective core of V1 is reflected in the fact that in our calculation the constraint expressed by eqs. (5) and (11) is automatically satisfied as an inequality throughout the entire density range displayed, and hence the parameter $\lambda$ is always zero for this case, as shown in table 1 . Our results lie somewhat above the HNC results, at worst by only about $10 \mathrm{MeV}$, and considering the excellent agreement obtained previously ${ }^{5,6}$ ) for the Betho homework problem (the repulsive 
part only of the potential V1), we believe this to be mainly due not to the higher order terms in the cluster expansion, but rather to the comparatively strong attractive part of this potential, which, at low densities, presumably acts to induce a small overshoot into the exact correlation function. We note however, that throughout the entire density range our results lie considerably closer to the presumably more exact HNC results than either of those obtained by the other lowest order methods of PLOCV or LOBT. The agreement also becomes more striking at higher densities.

By comparison the potential V2 has a larger core and a rather weak attraction. These facets of the potential are reflected in our calculation by the vital role that the constraint of eqs. (5) and (11) now plays in forcing the correlation function to heal, and hence constraining the neglected higher-order terms in the cluster series to remain small. Thus at a density of $\left(\frac{3}{2} \pi^{2} \rho\right)^{t}=2.0 \mathrm{fm}^{-1}$, the Lagrange multiplier $\lambda$ for this constraint has a value of $-104 \mathrm{MeV}$, which is over twice the magnitude of the maximum attraction in the potential. Other values are shown in table 1 , and it can be seen that the constraint does not operate (as an equality) until densities above $\left(\frac{3}{2} \pi^{2} \rho\right)^{+} \gtreqless 1.6 \mathrm{fm}^{-1}$. Below this density the natural healing distance $d$ is rather large due to the relatively weak attraction. Thus $d / r_{\mathrm{c}}=1$ at the rather low density $\left(\frac{3}{2} \pi^{2} \rho\right)^{\frac{7}{7}} \approx 1.1 \mathrm{fm}^{-1}$. Where the constraint starts to operate, the binding energy per particle already exceeds the HNC value by some $7 \mathrm{MeV}$, but for higher densities the constraint quickly ensures that the fractional difference between the present and the HNC results does not continue to rise. Thus although the difference in binding energies per particle is some $20 \mathrm{MeV}$ at saturation, the saturation densities themselves agree very well, and thereafter the two curves start to converge. By contrast the PLOCV saturation density is considerably lower, and the LOBT results have not saturated at all at considerably higher densities. Since the potential V2 clearly provides a very stringent test for any method, and since the HNC results should probably be regarded as at best an upper bound to the energy, we regard our results as being quite satisfactory.

The remainder of this section describes the results for Fermi systems of degeneracy $v=4$ (appropriate to nuclear matter). For fermions we have the additional choice as to the number of different correlacion functions employed, in that we may use different ones, for example, to describe even and odd parity states. Most of the model calculations with which we compare our results use just one state-independent correlation function in each case, and for the sake of valid comparison we also give results using one state-independent correlation function. However, we also display the results obtained using different functions for the even and odd parity states and, when appropriate (i.e., for the potential V4), by further subdividing the correlation functions to describe separately spin-singlet and spin-triplet states of each parity. In general, for the reasons cited in sect. 2, we use constraints (4)-(6) of the form given in eqs. (20)-(22), namely the normalization constraints; although for comparison we also show the results obtained using the Bose constraints (10)-(12).The results displayed in figs. 4-7 using our method with these two sets of constraints are labelled 


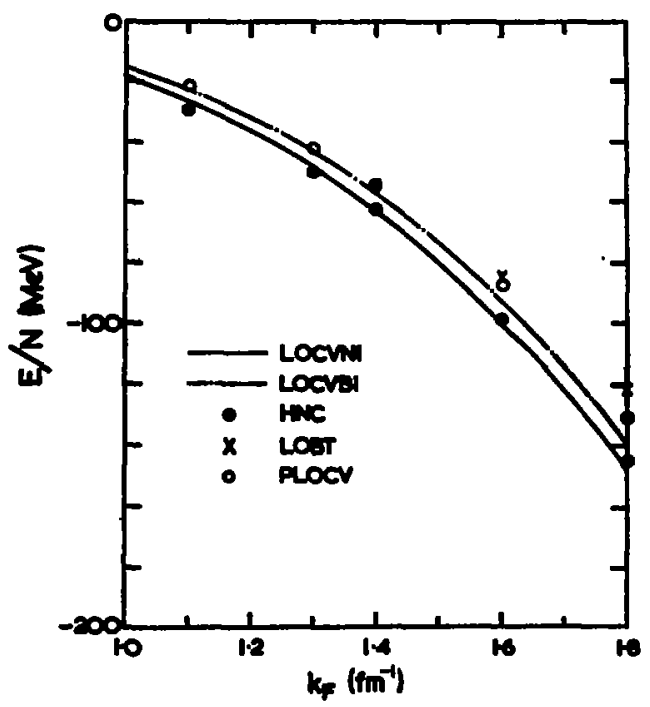

Fic. 4. The ground-state energy per particlo for a Fermi system $(v=4)$ with potential V1. The results of the present mothod using one state-independent correlation function and the normalization constraints or Bose constraints are labelled LOCVN1 and LOCVB1 respectively. The results using the hypernotted chain approximation (HNC), Pandharipande's lowest order constrained variation (PLOCV), and lowest order Brueckner theory (LOBT), are taken from rof. ${ }^{1}$ ).

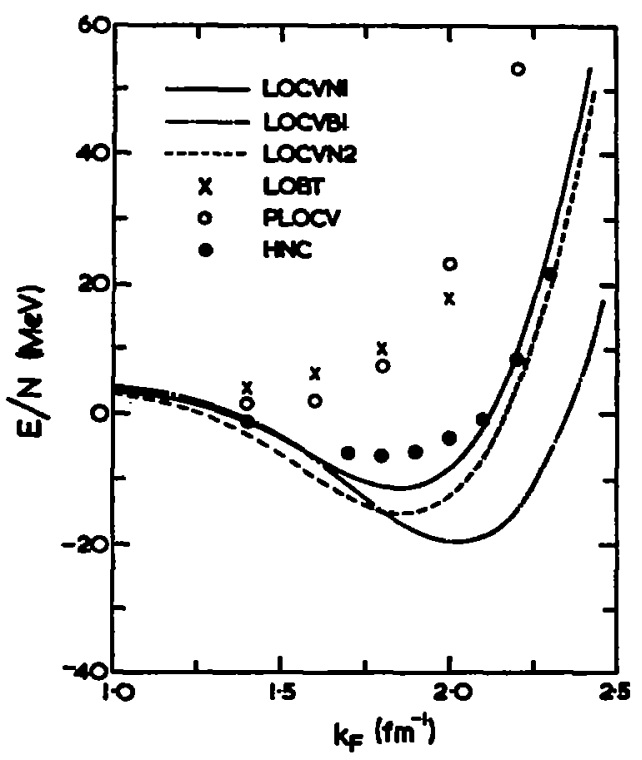

Fig. 5. Same as fig. 4, but with the potential V2. In addition tho curve LOCVN2 gives the results of the present method using the normalization constraints plus two independent correlation functions, one for each parity. 


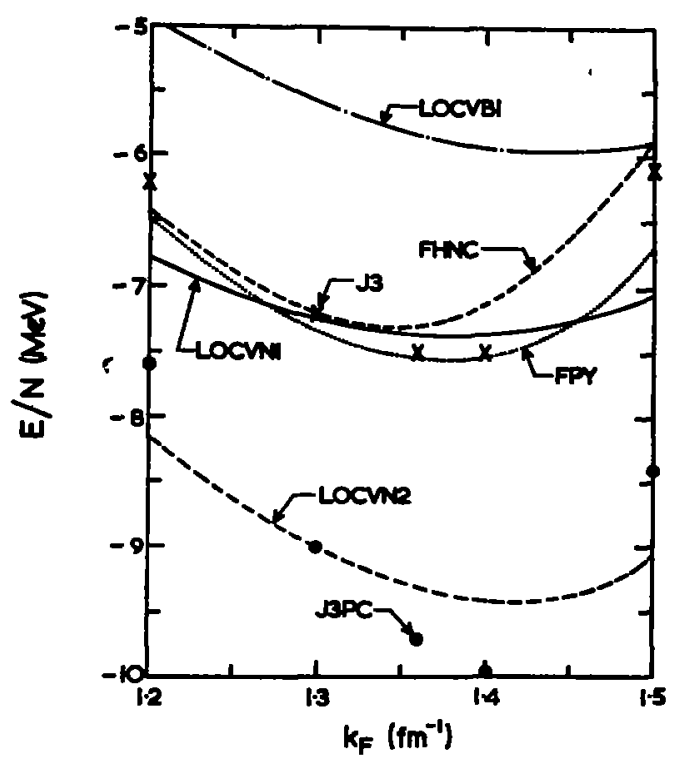

Fig. 6. The ground-state energy per particle for a Fermi system $(y=4)$ with potential V3. The results of the present method using one state-independent correlation function and the normalization constraints or Bose constraints are labelled LOCVN1 and LOCVB1 respectively; and with the normalization constraints with two independent correlations, one for each parity, as LOCVN2. The results using the fermion hypernetted chain (FHNC) and fermion Percus-Yevick (FPY) approximations are taken from rof. ${ }^{2}$ ); those from evaluating the throo-body Jastrow terms (J3) are taken from ref. ${ }^{13}$ ); and those from evaluating the second-order perturbation correction to J3 (J3PC) are taken from ref. ${ }^{3}$ ).

LOCVN and LOCVB respectively, and the additional number appended to these labels indicates the number of distinct correlation functions which we solve for variationally. Thus in the latter case we show only the results using one state-independent correlation function, which are accordingly labelled LOCVB1.

The fermion results for V1 shown in fig. 4 are very similar to the boson results for this potential, except that the small overshoot permitted in the correlation function with the normalization constraint brings us rather closer to the HNC results. The effect of allowing state dependence in the correlation function is too small to be displayed on the scale of fig. 4. Again it may be seen that our results lie closer to the HNC results for this potential than either of those obtained by PLOCV or LOBT methods.

By comparison with the boson results it is to be expected that the potential V2 will provide a much more stringent test of the effectiveness of our constraints. It is clear from fig. 5 that, as expected, the normalization constraint is superior to the Bose constraint, particularly at high densities. A striking feature is that the LOCVN1 results bear qualitatively the same relationship to the HNC results, with regard to both good agreement between the saturation densities and convergence of the two 


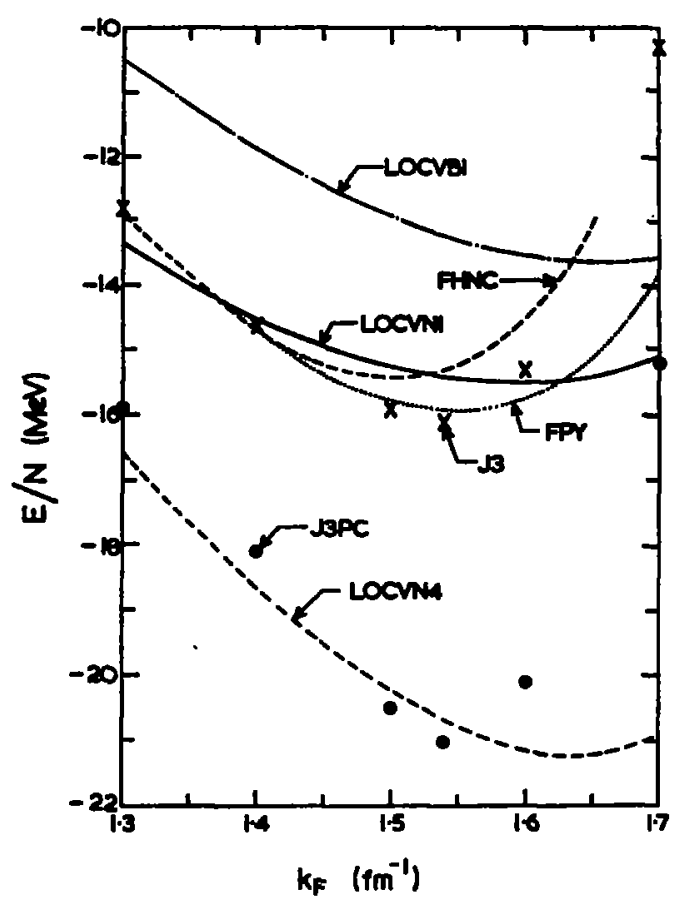

Fig. 7. Same as fig. 6, but with the potential V4, In addition the curve LOCVN4 gives the reaults of the present method using the normalieation constraints plus four independent correlation functions, ono for each combination of spin and parity.

results thereafter, as already described for bosons; and as a bonus the overall agreement is improved. By contrast the LOCVB1 results behave quite differently. We take this as evidence that the normalization rather than the Bose constraint is indeed the more natural extension to fermions of the constraint applied to bosons. In fig. 5 we also show the effect of allowing different correlation functions in even and odd parity states (the curve LOCVN2) and, even though the potential V2 is the same in both states, we see that it is possible thereby to lower the energy by about $3.5 \mathrm{MeV}$ at $k_{\mathrm{F}} \approx 1.8 \mathrm{fm}^{-1}$. This is a most significant result and could arise for one of two main reasons. We conclude that either method of constraining the correlation function becomes unreliable when we introduce state dependence into it, or this lowering of the energy is a genuine effect. We see no physical reason for the former to hold true, and the results obtained for the potentials V3 and V4 indicate that the latter may well be the main reason, as explained below. Indeed a similar effect is also observed in the HNC method ${ }^{15}$ ), which is the only other method with which we compare results that permits state dependence.

For the potentials V3 and V4 we compare our results in figs. 6 and 7 with those obtained from the many-body calculations of Campani et al. ${ }^{2}$ ) who describe two 
different approximations to the exact fermion pair distribution function, namely the fermion hypernetted chain (FHNC) and fermion Percus-Yevick (FPY) approximations. These authors perform a minimization of their final many-body approximation to the energy in each case by using a particular parametrization of the correlation function which contains no "overshoot", and varying the parameters to obtain the minimum. We also show in figs. 6 and 7 both the results for these potentials of Backman et al. ${ }^{13}$ ), labelled J3, who evaluate the energy up to and including the threebody Jastrow terms, and the recent results of Clark et al. ${ }^{3}$ ), labelled J3PC, who include a second-order perturbation correction to the results of ref. ${ }^{13}$ ) within the framework of the method of correlated basis functions. The results J3 of Backman et al. ${ }^{13}$ ) are obtained by minimization of the energy with respect to the parameters of their chosen form for the correlation function which does allow "overshoot". (These authors also impose a different constraint than either of those considered by us, namely the orthogonality constraint.)

We first observe from figs. 6 and 7 that for both potentials V3 and V4, the use of the normalization constraint in the context of our constrained variational method gives energies which are very close to those obtained using the more sophisticated approaches described above. In particular, the addition of our LOCVN1 results to the FHNC, FPY and J3 results, all of which also employ just one state-independent correlation function, nowhere significantly increases their overall spread. Secondly, we notice that imposing the normalization constraint again gives results which are clearly superior by comparison, to those obtained with the Bose constraint.

For the potential V3 in fig. 6 we show the effect of allowing a parity-dependent corr lation function (i.e., the curve labelled LOCVN2), whereby the energy is lowered by about $2 \mathrm{MeV}$ at $k_{\mathrm{F}}=1.4 \mathrm{fm}^{-1}$; and for V4 we show in fig. 7 the effect of allowing both parity and spin dependence in the correlation function (i.e. the curve labelled LOCVN4), whereby the energy is lowered by about $4 \mathrm{MeV}$ at $k_{\mathrm{F}}=1.4$ $\mathrm{fm}^{-1}$ and by about $6 \mathrm{MeV}$ at $k_{\mathrm{F}}=1.6 \mathrm{fm}^{-1}$. There is clearly qualitative agreement at the very least between these results and the results J3PC obtained by Clark et al. ${ }^{3}$ ). At the densities of interest, the effect of our normalization integral constraint is not too great for either potential V3 or V4, as may be seen by comparing the values of the parameter $\lambda$ (which is the Lagrange multiplier associated with the constraint) from table 1 with typical values of the potential from fig. 1 , in each case. This fact, when taken together with the results J3PC of Clark et al. ${ }^{3}$ ), indicates that the gain in energy we obtain in each case by allowing state-dependent correlation functions is a real effect, which would also be reflected in the more sophisticated calculations were they adapted to deal with state-dependent correlation functions. We also suggest that the significant second-order perturbation correction to the energy found by Clark et al., in passing from the results J3 to the results J3PC, is deceptively large, and is in large measure due to the fact that they used state-independent correlation functions in the minimization of their uncorrected energy. 


\section{Discusion and conclusions}

We show in fig. 8 results for the correlation functions obtained for the hard-core potential V3 for a system of fermions by our LOCVN method, using both one stateindependent function, and two functions (one for each parity). The curves are drawn for a Fermi momentum $k_{F}=1.36 \mathrm{fm}^{-1}$, but they are quite typical of the entire density range. For the potentials V3 and V4 it is interesting to note from figs. 6 and 7 that the small degree of overshoot allowed in the LOCVN correlation function (and see fig. 8) through constraint (18), has the effect of lowering the energy found by the LOCVB method almost exactly into line with the FHNC or FPY energies. These latter results, it should be remembered, have been calculated using correlation func-

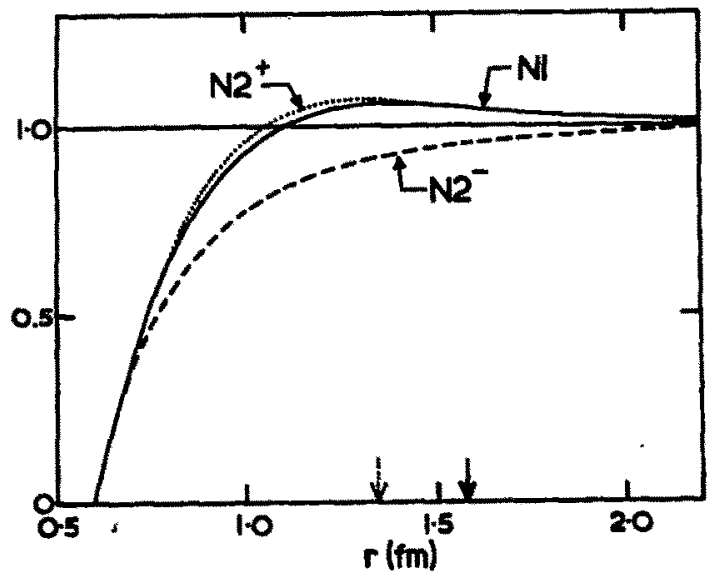

Fig. 8. The correlation functions obtained with the present method and the normalization constraints for a Fermi systom $(v=4)$ of Fermi momontum $k_{\mathrm{P}}=1.36 \mathrm{fm}^{-1}$, for potential V3. Results are shown using one state-independent correlation function (N1); and two correlation functions, one for positive $\left(\mathrm{N}^{+}\right)$and one for negative $\left(\mathrm{N}^{-}\right)$party states. The arrows indicate the corrolation distances, $d$; that for $\mathrm{N}_{2}-$ occurs off-scale at $r=2.88 \mathrm{fm}$.

tions without overshoot. A similar result is also seen to be true from fig. 4 for the potential V1, with respect to the HNC results; and similarly, from fig. 5, for the potential V2 for $k_{\mathrm{F}} \leqslant 1.5 \mathrm{fm}^{-1}$, although the effect here is much less marked due to the relative weakness of the attraction. The occurrence of this for four quite different potentials is unlikely to be coincidental, and is almost certainly related to the fact that when the cluster expansion is converging well, we may regard the expression $f^{2} \phi^{2}$ calculated in the LOCVN method as a good approximation to the exact pair distribution function. The exact distribution function will certainly overshoot since it must satisfy the exact normalization condition. In so far as we have incorporated this condition into our LOCVN method, it is to be expected that in some average sense we have about the right amount of total overshoot automatically built into our correlation function. We also note that in the work of 
Campani et al. ${ }^{2}$ ) (and see their table 1), allowing overshoot into their correlation functions resulted in only a very slight lowering of the energy; e.g. by only $0.1 \mathrm{MeV}$ in the FPY approximation for the potential V3 at $k_{\mathrm{F}}=1.36 \mathrm{fm}^{-1}$. It may very well be that provided the normalization condition is satisfied in even only a crude average sense, then the actual form for the overshoot is not very critical. In fact, it is clear that two competing demands on the correlation function are in play. For potentials of the form containing a strong short-range repulsion and a long-range attraction, the potential term in eq. (7) will demand a sharp overshoot, whilst the two-body kinetic term [proportional to $f_{l}^{\prime 2}(r)$ in eq. (7)] will demand a shallow overshoot. The interplay between these two opposing tendencies could very easily give rise to a broad spectrum of acceptable correlation functions, which could be distinguished only by focussing on some other quantity than the ground-state energy.

The results we have presented in the previous section have indicated that the method of lowest order constrained variation given in sect. 2 is capable of providing an excellent description of infinite systems of both bosons and fermions interacting through a wide variety of potentials that model the real internucleon force, and up to densities above that of nuclear matter. In particular, if we use just one stateindependent correlation function, we obtain close agreement in all cases with the much more ambitious and computationally more difficult methods already described that likewise employ a state-independent correlation function, but which attempt to go beyond lowest order. In all cases this agreement is better than with any of the other lowest order methods.

Finally, we have also seen that the use of state-dependent correlations can give considerably lower energies in our method, in apparent agreement with the indications found by Clark et al. ${ }^{3}$ ) in the entirely different context of using the method of correlated basis functions. A similar effect has also been observed by Pandharipande and Bethe ${ }^{15}$ ) using the HNC method with a state-independent potential suitable to liquid ${ }^{3} \mathrm{He}$. These authors find energies which are considerably lower than previous calculations, which they also ascribe to allowing angular variation and state-dependence in the correlation function. Within the context of a calculation of the effective mass of a ${ }^{3} \mathrm{He}$ atom in liquid ${ }^{4} \mathrm{He}$, Pandharipande and Itoh ${ }^{16}$ ) have attempted to connect this lowering of the energy effect with the backflow current. They argue that a spherically symmetric correlation function does not correctly incorporate the backflow, whereas this can be incorporated by allowing state dependence. In any event, from the results of our own calculations, we believe the present effects to be real, and we look forward with great interest to the appearance of other calculations which include state-dependent correlation functions with these model potentials, using the higher-order approximations, so that this may be more fully investigated.

\section{References}

1) V. R. Pandharipande, R. B. Wiringa and B. D. Day, Phys. Lett. S7B (1975) 205

2) E. Campani, S. Fantoni and S. Rosati, Nuovo Cim. Lett. 15 (1976) 217 
3) J. W. Clark, P. M. Lam and W. J. Ter Louw, Nucl. Phys. A255 (1975) 1

4) O. Benhar, C. Clofi Degli Atti, A. Kallio, L. Lantto and P. Toropainen, Phys. Lett. 60B (1976) 129

5) J. C. Owen, R. F. Birhop and J. M. Irvine, Phy, Lett. S9B (1975) 1

6) J. C. Owen, R. F. Binhop and J. M. Irvine, Ann. of Phys, to bo publishod

7) J. C. Owen, R. F. Bishop and J. M. Irvine, Phy. Lett. 61B (1976) 147

8) R. V. Reid, Ann. of Phys. 50 (1968) 411

9) F. Iwamoto and M. Yamada, Prok. Theor. Phyn. 18 (1957) 345

10) T. Ohmurg, M. Morita and M. Yamada, Prog. Theor. Phys. 15 (1956) 222

11) M. E. Grypeos and E. Mavrommati, Nwovo Cim. Lett. 5 (1972) 369

12) G. P. Mueller and J. W. Clark, Nucl. Phys. A15s (1970) 561;

M. L. Ristis, W. J. Ter Louw and J. W. Clark, Phys. Rov. C3 (1971) 1504

13) S.-O. Backman, D. A. Chakkalakal and J. W. Clark, Nucl. Phys. A130 (1969) 635;

S.-O. Buckman, J. W. Clark, W. J. Ter Louw, D. A. Chakkalakal and M. L. Ristig, Phys. Lett. $41 B$ (1972) 247

14) V. J. Emery, Nucl. Phys. 6 (1958) 585

15) V. R. Pandharipande and H. A. Bethe, Phys. Rev. C7 (1973) 1312

16) V. R. Pandharipande and N. Itoh, Phys. Rev. $A 8$ (1973) 2564 